From the Association of Departments of Family Medicine

Ann Fam Med 2004;2:281. DOI: 10.1370/afm.207.

\section{THE END OF NORMALCY}

There was nothing normal about the recent meeting of the Association of Departments of Family Medicine. The meeting itself was innovative, with presentations and discussions about new clinical models, including systems of care for communities and for employers; better methods of assessing faculty performance ${ }_{i}$ balancing clinical productivity and clinical education; international opportunities; and better communication with deans, faculty, and other members of the family medicine family. Special sessions were held on common management and leadership challenges for chairs, while other sessions focused on developing similar programs for faculty and on development opportunities. The Future of Family Medicine project was the subject of considerable thought and debate, including the need for more discussion within the "family" about the needs and concerns of academic departments and what departments can realistically achieve given the larger problems of our health care system.

Many departments expressed frustration and disappointment at declining student interest in the face of growing success in gaining curriculum time and placing faculty in dean's offices. There was agreement, however, that student interest in a discipline is not the only sign of departmental achievement. The clinical volume and productivity of the faculty, number of grants awarded and articles published, number of students and classes taught, and student evaluations are more common standards by which departments are judged. On these criteria, many departments are doing quite well.

Clinical office redesign is turning into a topic of interest, partially because of the work of the Institute for Healthcare Improvement, with which many departments participate, but also because of growing interest across schools to find ways to deliver better, safer care. Organizing and packaging primary care services in ways that are attractive to local employers and insurers is a theme being pursued by several departments.

Research within family medicine departments is growing, with more than 10 departments receiving $\$ 1$ million or more each year in NIH support, while the reported total NIH funding to family medicine departments is just less than $\$ 40$ million. The number of departments with substantial $\mathrm{NIH}$ support is undoubtedly much higher, as schools are inconsistent in coding primary departments.
The innovations at the meeting were not restricted to the departments themselves. The ADFM membership also approved revisions in the membership criteria, including full membership for some chairs of osteopathic departments, as well as some satellite and regional campus chairs, who share the challenges of working within the full scope of academic medicine. In addition, ADFM is pursuing hiring its first executive director, added standing committees, and shortened the term of the president, so that more members can serve in leadership capacities. If there is a shadow to the growing stature of the academic departments, it is in the small but increasingly visible differences between the departments and the wider discipline. In part, this divergence reflects the maturity of the discipline, as well as of the departments themselves, and of our ability to respectfully hold different opinions and perspectives. But it also represents a potential fault line that could expand into a town-gown divide. Departments must adapt to institutional expectations, even at the risk of creating stress with their practicing colleagues. But unthinking adaptation can lead to loss of the core social mission of family medicine and the opportunity for needed change in how students and residents are taught and how patients are cared for. The norm for ADFM meetings will likely continue to be exploration of innovative clinical, research, and educational programs; continued celebration of departmental successes; and continued discussion about ways in which we can work together to improve the care of all.

Lloyd Michener, MD Duke University

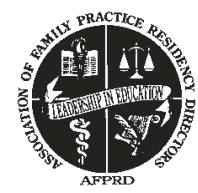

From the Association of Family Practice Residency Directors

Ann Fam Med 2004;2:281-282. DOI: 10.1370/afm.208.

\section{TEACHING TOMORROW'S LEADERS, TODAY}

As family medicine educators, it is our responsibility to teach the content and culture of our specialty to the next generation of family physicians. The publication of the Future of Family Medicine (FFM) report provides a landmark document for residency directors to help fulfill that responsibility. At an organizational level, the Association of Family Practice Residency Directors (AFPRD) has committed itself to leading the implementation of the FFM recommendation concerning family medicine education.

FFM calls for a period of innovation and experimentation in residency education. It reemphasizes the 
specialty's commitment to the full continuum of health care, including maternity and hospital care, in addition to community- and office-based practice. Furthermore, it challenges our residencies to maintain the core values of our specialty while embracing new technologies in the delivery of health care, such as electronic health records. Finally, the call for a new model of family medicine pushes our residency programs to totally reexamine our family practice centers from the way we schedule and communicate with patients to the services we offer. The family practice center was one of our specialty's major educational innovations 35 years ago. Now is the time to be innovative in the family practice center once again.

The AFPRD has embarked on a number of initiatives to contribute to the implementation of FFM. First, the organization's membership will be voting on a proposal to change our name to the Association of Family Medicine Residency Directors (AFMRD) at our June 2004 business meeting so we are consistent with the FFM recommendation on communication. To further the leadership and advocacy recommendation, the National Institute for Program Director Development (NIPDD) Academic Council is developing an advanced course on leadership for our membership. We are also initiating an awards program to recognize the hard work and leadership already shown by residency directors. To enhance residents' understanding of chronic and preventative medical care services discussed in the new model of family medicine, we are currently offering the Better Bones conferences on osteoporosis, and a similar series on diabetes is being planned for 2005 . AFPRD is supporting the recommendation on enhancing the science of family medicine by working with the North American Primary Care Research Group (NAPCRG) to promote research within our residencies.

Finally, at this year's program directors' workshop, there will be a plenary session devoted to FFM and 2 discussion forums to promote innovation in family medicine residency education. One forum will provide input into the proposed RRC revisions for residency accreditation, and the second will be on the Family Medicine Curriculum Resource Project and developing a standardized residency curriculum for the future.

Our current residents will ultimately be leading the implementation of the FFM recommendations during their practice careers. The AFPRD is fully committed to making the changes needed today to ensure that our residents are well prepared to be tomorrow's leaders.

Robin O. Winter, MD, MMM, CPE, FACPE President, AFPRD

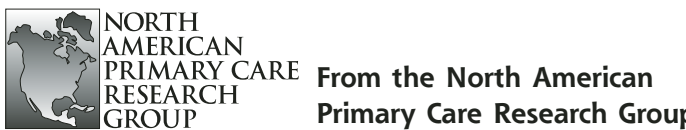

Ann Fam Med 2004;2:282-283. DOI: 10.1370/afm.203.

\section{FUTURE OF FAMILY MEDICINE RECOM- MENDATIONS CONFIRM NEED FOR INCREASED RESEARCH FROM FAMILY PHYSICIANS}

Members of NAPCRG and others might scan the Future of Family Medicine (FFM) report ${ }^{1}$ and conclude that research has been neglected, with only 1 of 10 recommendations clearly focused on the science of family medicine. They would, however, be wrong. This family medicine report is laced with research that predated its beginning, continued through the current ignition point, and will continue on into the foreseeable future.

FFM spent most of its money on research and based its deliberations and conclusions on research results from all over the world. As a set of compass headings, compared with an exact blueprint, the FFM report declares that further research is a necessity to guide a serious revision of family medicine. The report calls early and often for various types of research, especially effectiveness research, because, "These ideas need to be tested in practice."

Among 5 key challenges facing family medicine, 2 particularly call out for NAPCRG's assistance: (1) addressing the public's perception that family medicine is not solidly grounded in science and technology, and (2) winning respect in academic circles. Indeed, just as the report concludes that "the problems afflicting family medicine do not include irrelevance or obsolescence," the same can be safely concluded for the research enterprise so dear to NAPCRG

The basket of services expected of all family physicians includes quality improvement and practice-based research. The new model of practice is contrasted with the old model in that the old model consumed knowledge, but the new model will both consume and produce knowledge. The report calls for further development of practice-based research networks and sentinel practice systems and, with remarkable clarity, a reconciliation between family medicine and academic health centers. Even the identity statement formulated for family physicians acknowledges the role of science in family medicine.

Family medicine residency training of the future is expected in this report to require a "culture of innovation and experimentation. The educational process must train family physicians who can function optimally in the New Model practice ... who actively mea- 